\title{
Optimal Routing for Off-Hour Delivery Trucks with Noise- Reduction and Transport Costs Objectives
}

\author{
Yang Wang, Shanshan Wei, Yanyan Chen* \\ Beijing Key Laboratory of Traffic Engineering, Beijing University of Technology, Beijing, China 100012. \\ * Corresponding author. Tel.: 861-6739-1680; email: cdyan@bjut.edu.cn \\ Manuscript submitted April 16, 2019; accepted August 8, 2019. \\ doi: $10.17706 /$ jcp.14.10.615-623
}

\begin{abstract}
The off-hours delivery has been promoted as a mean to alleviate the traffic congestion and emission. However, such strategy can cause noise pollution to the residents who are close to the roads. To reduce such noise impact and transport costs simultaneously, this work intends to develop an algorithm to plan a route for delivery trucks to avoid the noise sensitive areas as much as possible while reduce transport costs. By considering the characteristics of land use, an algorithm to determine the noise vulnerability degree for each road link is developed in this paper. With the network associated with noise vulnerability degree and transport costs for each link, the routing problem is formulated as a bi-objective optimization problem, which can be easily solved by conventional approaches. Finally, to demonstrate the capability of the proposed algorithm, a simple delivery scenario has been taken and the comparison to the optimal route generated with single objective are made.
\end{abstract}

Key words: Off-hour delivery, optimal routing, traffic noise, noise reduction.

\section{Introduction}

With the rapid development of e-commerce, freight transport is expected to grow in the coming years at a faster rate than ever before [1]. This implies that the current worrisome traffic situation in urban areas could be further deteriorated as urban trucking during peak hours largely contributes to the traffic congestion. To remedy this issue, the reduction of freight vehicle trips during peak hours has been a common policy goal with the rapid development of logistics industry in recent years [2]. The off-hours delivery (OHD) strategy has been extensively investigated from various aspects based on a number of pilot projects in a number of cites [3], [4]. With the success of the pilot projects, it is evident that the OHD strategy can greatly result in a reduction in traffic congestion and emission [4]. Furthermore, the number of trips can be reduced if the consolidation strategy is implemented simultaneously with the assistance of large-size trucks during offhours [5]. However, it should be aware that noise nuisance may arise from OHD if trucks travel around noisesensitive areas, such as hospitals or residential communities.

In recent years, noise pollution has become one of the major factors affecting the quality of life in urban areas worldwide [6]-[8]. Excessive exposure to noise pollution not only seriously affects stress-related mental health, but also increases the risk of diseases such as ischemic heart disease as well as sleep disturbance, cognitive impairment among children, annoyance, and tinnitus [9]-[12]. In addition, continuous complaints on noise have been made in recent years. According to China Environmental Noise Prevention and control Annual Report, the country received a total of 520,000 environmental noise complaints, accounting for $43.9 \%$ 
of the total environmental complaints in 2016 [13]. In recent years, traffic noise is becoming one of the most prominent source of noise pollution in urban areas with the rapid growth of the vehicle ownerships. Consequently, the control of traffic noise has become a critical issue.

Many studies have investigated the traffic noise mitigation methods, with a particular focus on noise assessment [14], [15], traffic noise model [16], and noise mapping [17], [18]. However, the spatial distribution of noise pollution in urban areas is related to various factors, such as the building density, the shape and physical position of buildings, the type of channel and the distribution of the population, etc. [19]. In order to guarantee the quality of sound environment for the daily life of urban residents, the Standard of Environmental Noise of Urban Area (GB3096-2008) was issued in 2008. The regulatory standards were formed by dividing an urban area into a number of noise control zones and each has a designated noise limit which was developed based on the function characteristics of land use and the environmental quality requirements [20].

Table 1 lists the upper thresholds of environmental noise for different zones. It can be seen that the noise control standards during the day and night are different, and the maximum noise limit for a zone at night is lower than that in the daytime. Fig. 1 shows the rates that the noise intensity levels collected from 319 cities during 2016 are below the noise limits presented in Table 1 [13]. Obviously, the qualification rate for the class 4a (i.e., the areas next to the road sides) during the nighttime is the lowest, indicating traffic noise pollution is still serious. This implies that the shift to OHD can aggravate the noise pollution for areas close to the road sides due to the fact that the noise produced by a truck is much larger than a passenger car in general. Using light vehicles or other vehicular technology is one way to reduce the generated noise intensity level. However, the work, as part of our ongoing project, presented in this paper seeks a different way to reduce the noise nuisance by managing the delivery routes. From the perspective of social benefit and operator costs, this paper aims to develop an algorithm to plan a route for delivery trucks to avoid the noise sensitive areas as much as possible and reduce transport costs simultaneously.

Table 1. Noise Limits for the Classified Areas in China dB(A)

\begin{tabular}{|c|c|c|c|c|}
\hline \multicolumn{2}{|c|}{ Zone classification } & Description & Daytime & Nighttime \\
\hline \multicolumn{2}{|c|}{ Class 0} & Particularly protected areas & 50 & 40 \\
\hline \multicolumn{2}{|c|}{ Class 1} & Residential area & 55 & 45 \\
\hline \multicolumn{2}{|c|}{ Class 2} & Mixed area & 60 & 50 \\
\hline \multicolumn{2}{|c|}{ Class 3} & Industrial area & 65 & 55 \\
\hline \multirow{2}{*}{ Class 4} & a & Both sides of road traffic area & 70 & 55 \\
\hline & $\mathrm{b}$ & Both sides of railway area & 70 & 60 \\
\hline
\end{tabular}

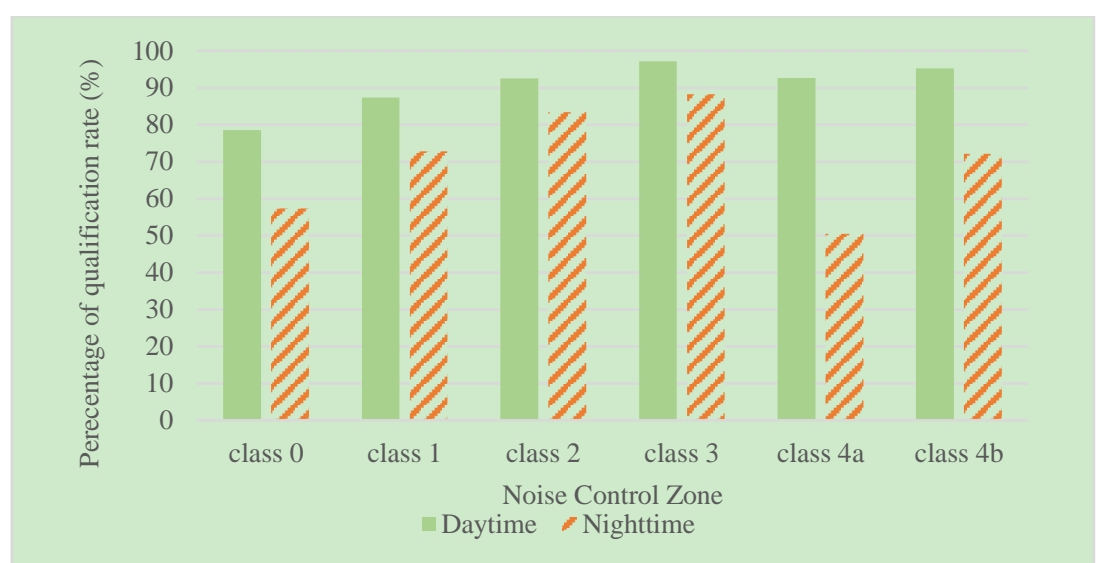

Fig. 1. The qualification rates of different noise control zones. 
This paper is structured as follows: the next section describes the detail of the optimal routing algorithm with the noise and the transport costs reduction objectives. In Section 3, scenario is presented for a location in Beijing, as a typical example to demonstrate the feasibility of the routing algorithm. Section 4 discusses the experimental results and then concludes the paper.

\section{Problem Description and Model Formulation}

This study proposes a method for the shippers to determine the delivery routes in the nighttime with the consideration of the social environmental benefits and operator benefits. The algorithm proposed in this paper aims to mitigate noise impact and reduce transport costs simultaneously. By dividing the types of street buildings, giving each land type different weighting coefficients of noise sensitive in night, and getting the length of each type of land, the effect of noise caused by trucks passing through a road link can be estimated through the algorithm which presented in the following section. In addition, from the perspective of operator, the transport costs include labor cost (working hours), fuel consumption, vehicle maintenance and other expenses. Labor cost and fuel consumption are the two major factors related to transport costs that should be considered by shippers when making route selection.

\subsection{The Framework of the Proposed Algorithm}

As shown in Fig. 2, the optimization algorithm for managing the delivery route proposed to reduce noise nuisance and control transport costs includes the following steps. First of all, the classification for the noise control zones from the Standard of Environmental Noise of Urban Area is quite general which no classification of street buildings separately. Hence, the acoustic environment function area of street buildings is first divided into more detailed categories in this paper, based on their sensitivity to nighttime noise. Then, the weight of each area is determined through questionnaire method. Furthermore, the transport costs and the noise vulnerability of each road segment are estimated by the model proposed in this paper, through estimation of the length of different noise control zones on either side of each segment. Finally, the Dijkstra algorithm is used to determine the optimal route according to different objectives.

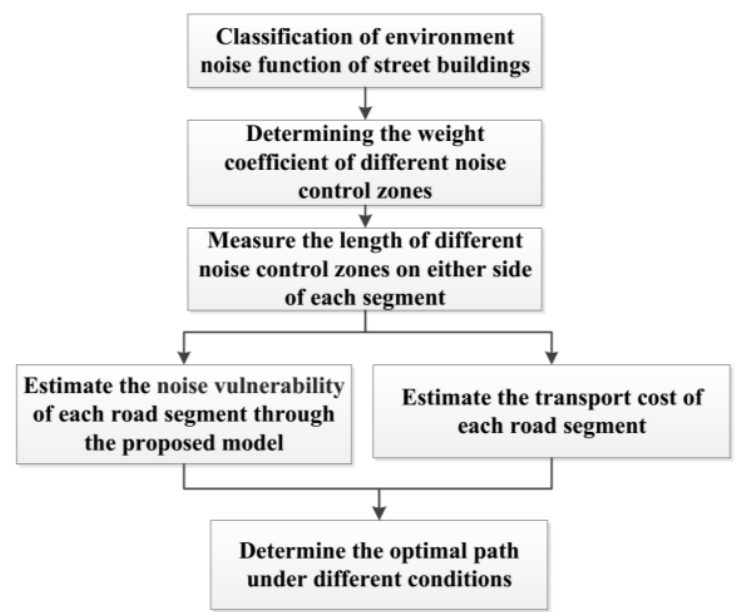

Fig. 2. The procedure to determine an optimal route with multiple objectives.

\subsection{Methodology}

\subsubsection{Classification of street buildings}

It should be aware that the zones of class 0 3 in in China Environmental Noise Prevention and control Annual Report are not specific to the areas along roadsides. However, the relative vulnerability of noise can 
be used for reference to determine the classification for street buildings. As shown in Table 2, in order to study the sensitivity of different types of street buildings to night noise, a more detailed classification of street buildings has been made based on the noise limits of various noise control zones of the Standard of Environmental Noise of Urban Area (GB3096-2008). They are divided into 7 types according to their sensitivity to night noise. Class1 is the areas that especially needed for quiet at night, such as sanatorium, hospital, senior hotel, etc. Class 2 and class 3 are the areas where a quiet environment is needed at night, such as residence, school, scientific research area, and office district. Class 4 is a mixed area that contains residential, commercial, industrial, etc. Class 5 is the area where the commercial area accounts for more than $70 \%$. Class 6 is the area where the industrial area accounts for more than $70 \%$. Class 7 is the area that is rarely affected by noise at night, such as parks, green spaces, squares and other areas that are basically unmanned at night.

Table 2. Classification of Street Buildings Based on Noise Sensitivity

\begin{tabular}{cc}
\hline \hline Zone classification & Description \\
\hline Class 1 & hospital, sanatorium, senior hotel \\
Class 2 & residence, \\
Class 3 & school, scientific research area, office district \\
Class 4 & the mixed areas \\
Class 5 & commercial area \\
Class 6 & industrial area \\
Class 7 & parks, green spaces, squares \\
\hline \hline
\end{tabular}

\subsubsection{Weight coefficient}

Instead of using the decibel limits listed in Table 1 for each classified zone, a questionnaire survey was conducted in order to get more objective weight coefficient. The respondents included those with different sex, age, occupation, etc., but the relationship between personal characteristics and noise sensitivity is not discussed in this paper.

The most important part of the questionnaire was that respondents scored different noise zones using the 10-point Likert scale to assess the sensitivity of each type of noise zones to noise in night, so as to determine the final weight coefficient. Relevant research shows that when the content of the topic in the questionnaire needs to obtain more accurate data, it can be controlled by means of the rank of the Likert scale. The more the magnitude, the more accurate the survey results, but the survey results will be further "high scores". This bias can be eliminated by reversing the order of scores [21]. Hence, the questionnaire of this paper, the score is also in the reverse order.

Table 3. The Results of Cronbach Reliability and Validity Analysis

\begin{tabular}{ccccc}
\hline \hline Class & CITC & The $\alpha$ that has been deleted & Cronbach $\alpha$ & KMO \\
\hline Class1 & 0.574 & 0.756 & & \\
Class 2 & 0.439 & 0.779 & & \\
Class 3 & 0.582 & 0.752 & 0.791 & 0.740 \\
Class 4 & 0.625 & 0.746 & & \\
Class 5 & 0.571 & 0.755 & & \\
Class 6 & 0.424 & 0.782 & & \\
Class 7 & 0.468 & 0.781 & & \\
\hline \hline
\end{tabular}

The results of the investigation show that a total of 214 people conducted the survey, including 193 valid questionnaires and 21 invalid questionnaires. The reliability and validity test results of the questionnaire are shown in Table 3. The CITC is the corrected item-total correlation. The Cronbach $\alpha$ is a statistic, which refers 
to the average value of the half-confidence coefficient obtained by all possible item partitioning methods of the scale, it's the most commonly used reliability measurement method. The KMO is used to compare simple correlation coefficient and partial correlation coefficient between variables. According to the above analysis, the results of the questionnaire survey have good credibility, and then the average scores of each class are obtained, the final weight is obtained and the results are shown in Table 4.

Table 4. The Weight of Each Class

\begin{tabular}{cccccccc}
\hline \hline Class & 1 & 2 & 3 & 4 & 5 & 6 & 7 \\
\hline Weight & 0.23 & 0.19 & 0.17 & 0.14 & 0.11 & 0.09 & 0.07 \\
\hline \hline
\end{tabular}

\subsubsection{Determination of the noise vulnerability for a road link}

When the impact of vehicle noise is considered from the origin to the destination, the noise vulnerability for a road link is determined as follows: firstly, the length of each class noise control zone on the both sides of the road link is obtained which can be measured via the road network. Secondly, the noise vulnerability for the road link is estimated by aggregating each zone length with the weights of zones, as shown in (1).

$$
N_{i}=\sum_{k=1}^{k=7} l_{i k} w_{k}
$$

where, $N_{i}$ is the noise vulnerability of road link $i, l_{i k}$ is the zone length of class $k$ of $\operatorname{road} \operatorname{link} i, w_{k}$ is the weight coefficient of class $\mathrm{k}$ of road link $i$ which is given in Section 2.2.2.

\subsubsection{Estimate the transport costs of each road segment}

The transport costs considered in this paper mainly have the following two aspects: labor cost (working hours) and fuel consumption. To simplify the calculation, travel time and route length are used to estimate the approximate monetary value of working hours and fuel consumption, respectively. As shown in (2), the cost of working time is a function of travel time which can be obtained by dividing the route length over free flow speed which is generally true due to light traffic during nighttime. In this paper, we used the speed limit to present the free flow speed. Formula (3) and (4) show the calculation of fuel cost and total transport costs.

$$
\begin{gathered}
M_{T i}=L_{i} / v_{i}^{*} \lambda \\
M_{L i}=L_{i}^{*} \beta^{*} \varepsilon \\
M_{i}=M_{T i}+M_{L i}
\end{gathered}
$$

where, $M_{T i}$ is the cost of travel time when the vehicle passes through link $i . L_{i}$ is the length of road link $i . v_{i}$ is the upper speed limit of road link $i . \lambda$ is the conversion coefficient of time, which is equal to the local hourly wage level. According to the latest announcement of the minimum wage standard for Beijing in 2018, the salary standard for full time and part time workers is taken into account, $\lambda=19$ RMB yuan/hour is adopted in this paper. $M_{L i}$ is the cost of fuel consumption when the vehicle passes through link $i . \beta$ is the conversion coefficient of route length, and its value depends on the fuel consumption per hundred kilometers of the truck. Based on previous studies: compliance with GB/T 12534-1990, the 40 diesel trucks required by the General Rules of Automobile Road Test Method for the preparation of sample vehicles are tested, the rated 
power is $50 \sim 368 \mathrm{kw}$, the displacement is $1.5 \sim 13.0 \mathrm{~L}$, the test quality is $2.17 \sim 49.00 \mathrm{t}$, the test vehicle is fully loaded, and the fuel consumption results are 7.17 36.52 L/100km [22]. This paper takes the average value of test result, $\beta=22 \mathrm{~L} / 100 \mathrm{~km}$ in this paper. $\varepsilon$ is the price of diesel oil and it's not constant, oil prices vary in different regions and at different times. This paper takes the oil value $\varepsilon=6.95 \mathrm{RMB}$ yuan/ L in this paper.

\subsubsection{Optimal path selection}

There are many algorithms for choosing the optimal path, such as Dijkstra, Floyd, etc. It has been widely recognized that the Dijkstra's algorithm is one of the most efficient algorithms that can guarantee to find a global shortest route when all the weights are non-negative [23], [24]. Therefore, we have adopted the Dijkstra's algorithm in the current work in order to find the optimal routes with the objectives to reduce noise nuisance and transport costs.

\section{Case Study}

\subsection{Delivery Scenario}

To demonstrate the algorithm proposed for the truck routing at night, a simple scenario of Beijing has been considered in this work. The delivery task starts from the Yuegezhuang vegetable wholesale market to the Wu Mart Supermarket in Focus place, which are labeled as "O" and "D" in Fig. 3, respectively. The road network has been extracted to exclude any routes which can result in a significant detour. Figure 4 illustrates the extracted network with 92 links in total. Various types of roads, e.g., expressways, arterial roads, branch roads, etc., are included in the road network, while the seven noise control zones, i.e., particularly protected areas, residential, mixed area, etc., are along the roads in the network.

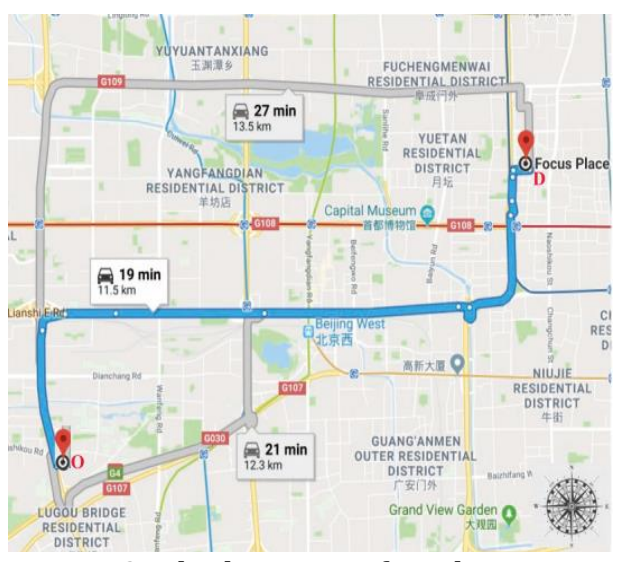

Fig. 3. The location of study area.

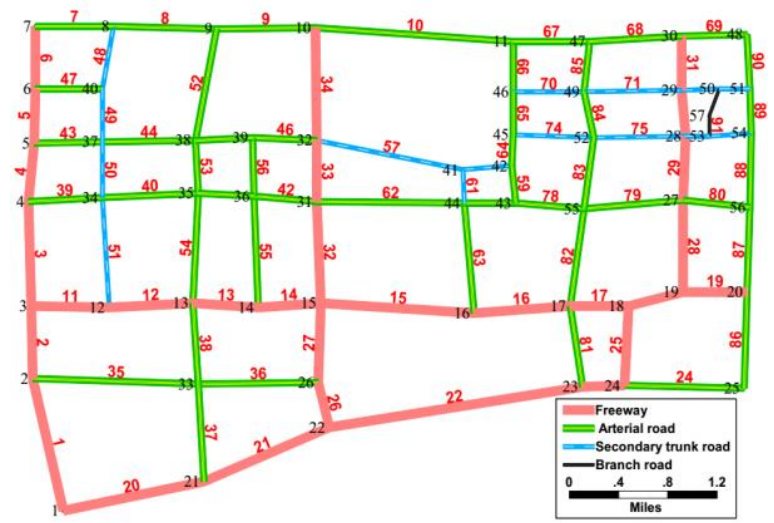

Fig. 4. Road network of study area.

\subsection{Result and Discussion}

\subsubsection{The results of path selection considering single factor}

To demonstrate the routing algorithm which can reduce noise nuisance, the minimum cost of fuel consumption and time are also taken as the respective objective to find an optimal path by the Dijkstra's algorithm. Fig. 5 (a)-(b) show the optimal paths (marked using arrows) for the objective to minimize transport costs and noise nuisance for the same network with each link associated with the transport costs and the noise vulnerability degree, respectively. Note that the transport costs for a route is obtained simply by summing the link transport costs, the noise vulnerability degree is determined by the algorithm presented in 2.2 .

Obviously, the optimal paths are different for the respective objectives, although both transport costs and noise nuisance take account of route length. With the aim to reduce transport costs, the roads with high speed 
and short length tend to be chosen. Similarly, the route obtained with least noise impact avoids the noisesensitive areas, such as hospitals or residential communities, as much as possible.

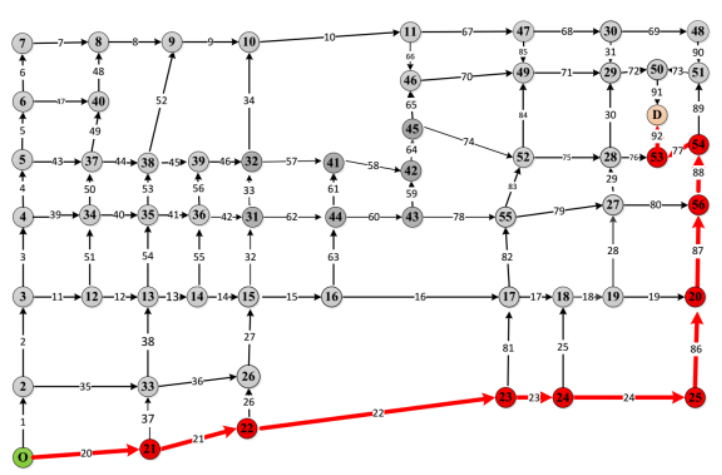

(a) Based on transport costs

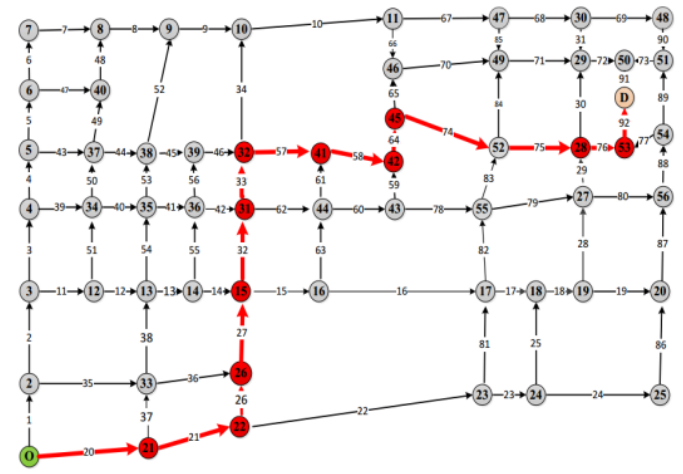

(b) Based on noise nuisance

Fig. 5. The optimal route for the OD with the different objectives.

\subsubsection{The results of path selection considering two factors}

In the actual night delivery process, it should consider how to reduce not only noise nuisance, but also the cost of operators. Therefore, it is very important to choose a delivery route which can reduce noise nuisance and transport costs. Fig. 6 show the optimal paths consider both transport costs and noise nuisance simultaneous.

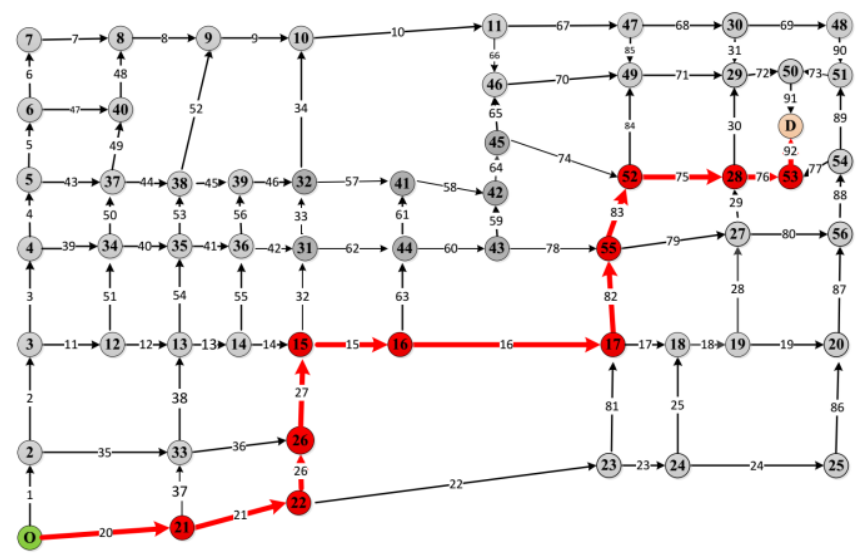

Fig. 6. The optimal route for the OD under double objectives.

\section{Conclusion}

This paper describes our work on the ongoing project aiming to reduce the noise nuisance to the local residents generated by the delivery trucks during nighttime while minimizing transport costs. Firstly, the acoustic environmental functional areas are classified more carefully, and their weights are determined by questionnaire survey. Then, an algorithm has been proposed to determine the noise vulnerability degree for each road link by assessing the potential areas influenced by the traffic noise for each type of noise control zones along the roadsides. With the formulation of the objective, the Dijkstra's algorithm is adopted to generate the best route under the objective. Finally, a simple delivery scenario is taken to demonstrate the capability of the proposed algorithm to effectively reduce the noise nuisance and transport costs with the comparison to the other routes obtained with the minimum noise nuisance, travel time and distance, respectively. Although the scenario exemplified in this study is simple, it is appropriate to explain the 
fundamental principle of the algorithm proposed in this paper.

However, the current work ignores the followings: the road noise level resulted from other trucks are not considered at the moment; a more realistic delivery task with more stops for a trip (which can be modelled as a travelling salesman problem) should be considered; different types of trucks may have different impact of noise. In addition to those research tasks, the future work will be also taking into account the distance from the street building to the road, the number of residents in the street building and the sensitivity of residents to noise at different time periods.

\section{Conflict of Interest}

"The authors declare no conflict of interest".

\section{Author Contributions}

The authors confirm contribution to the paper as follows: study conception and design: Yang Wang, Yanyan Chen; data collection: Shanshan Wei; analysis and interpretation of results: Shanshan Wei; draft manuscript preparation: Shanshan Wei. All authors reviewed the results and approved the final version of the manuscript.

\section{Acknowledgment}

The project has been funded by National Key R\&D Program of China (2016YFE0206800), Scientific Research Common Program of Beijing Municipal Commission of Education (KM201710005030), and the Foundation Research Fund Project of Beijing University of Technology (038000514315501).

\section{References}

[1] Mommens, K., Lebeau, P., Verlinde, S. et al. (2018). Evaluating the impact of off-hour deliveries: An application of the transport agent-based model. Transportation Research Part D Transport \& Environment, 62, 102-111.

[2] Sathaye, N., Harley, R., \& Madanat, S. (2010). Unintended environmental impacts of nighttime freight logistics activities. Transportation Research Part A: Policy and Practice, 44(8), 642-659.

[3] Holguín-Veras, J., Wang, C., Browne, M. et al. (2014). The New York city off-hour delivery project: Lessons for city logistics. Procedia - Social and Behavioral Sciences, 125, 36-48.

[4] Holguín-Veras, J., Encarnación, T., González, C. et al. (2016). Direct impacts of off-hour deliveries on urban freight emissions. Transportation Research Part D Transport \& Environment, 61, 84-103.

[5] Regue, G. R., \& Bristow, A. L. (2011). An exploration of the potential feasibility of a freight tram scheme in Barcelona. Proceedings of European Transport Conference.

[6] Frei, P., Mohler, E. et al. (2014). Effect of nocturnal road traffic noise exposure and annoyance on objective and subjective sleep quality. International Journal of Hygiene \& Environmental Health, 217(2-3), 188-195.

[7] Cristian, C., \& Cristina, P. (2016). A study of relationships between traffic noise and annoyance for different urban site typologies. Transportation Research Part D, 122-133.

[8] Hunashal, R. B., \& Patil, Y. B. (2012). Assessment of noise pollution indices in the city of Kolhapur, India. Procedia-Social and Behavioral Sciences, 37(1), 448-457.

[9] World health organization. Burden of disease from environmental noise. WHO and JRC.

[10] Abbaspour, M., Karimi, E., Nassiri P. et al. (2015). Hierarchal assessment of noise pollution in urban areas-A case study. Transportation Research Part D, 34, 95-103.

[11] Basner, M., Babisch, W., Davis, A. et al. (2014). Auditory and non-auditory effects of noise on health. Lancet, 383(9925), 1325-1332.

[12] Clark, C., Head, J., \& Stansfeld, S. A. (2013). Longitudinal effects of aircraft noise exposure on children's health and cognition: A six-year follow-up of the UKRANCH cohort. Environmental Psychology, 35, 1-9. 
[13] Ministry of Ecology and Environment of the People's Republic of China. China Environmental Noise Prevention and Control Annual Report.

[14] Buratti, C., Belloni, E., \& Moretti, E. (2014). Facade noise abatement prediction: New spectrum adaptation terms measured in field in different road and railway traffic conditions. Applied Acoustics, 76, 238-248.

[15] Lee, E. Y., Jerrett, M., Ross, Z. et al. (2014). Assessment of traffic-related noise in three cities in the United States. Environmental Research, 132, 182-189.

[16] Kumar, P., Nigam, S. P., \& Kumar, N. (2014). Vehicular traffic noise modeling using artificial neural network approach. Transportation Research Part C Emerging Technologies, 40, 111-122.

[17] Cai, M., Zou, J., Xie, J. et al. (2015). Road traffic noise mapping in Guangzhou using GIS and GPS. Applied Acoustics, 87, 94-102.

[18] Zuo, F., Li, Y., Johnson, S. et al. (2014). Temporal and spatial variability of traffic-related noise in the city of Toronto, Canada. Science of the Total Environment, 472(472C), 1100-1107.

[19] Ariza-Villaverde, A. B., Jiménez-Hornero, F. J. et al. (2014). Influence of urban morphology on total noise pollution: Multifractal description. Science of the Total Environment, 472, 1-8.

[20] China, M.o.E.P.o.t.P.s.R.o. Standard of Environmental Noise of Urban Area.

[21] Zhou, Q. Y., \& Chen, H. L. (2018). The law of psychological tendency of the respondents in statistical survey and its application. Statistics and Decision, 3, 34-38.

[22] Zhang, Z. B., Zheng, Y. Y. et al. (2018). Effect of quality on unit quality fuel consumption of operating freight cars. Beijing Automotive Engineering, 2.

[23] Dijkstra, E. W. (1959). A note on two problems in connexion with graphs. Numerische Mathematik, 1,269271.

[24] Chen, Y. Z., Shen, S. F., Chen, T. et al. (2014). Path optimization study for vehicles evacuation based on Dijkstra algorithm. Procedia Engineering, 71(C), 159-165.

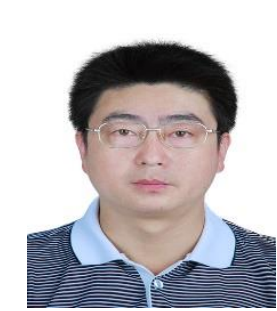

Yang Wang is currently an associate professor in the College of Metropolitan Transportation and Beijing Key Laboratory of Traffic Engineering, Beijing University of Technology. After obtaining his Ph.D degree from Loughborough University, he worked as a research associate at University of Portsmouth before moving to Beijing University of Technology. His current research centers on the areas of the urban fright transportation and the application of innovative methodologies to address the sustainability issues related to the urban transportation system including both freight and passenger transportation. 